\title{
Can cognitive behavioral therapy (CBT) intervention in patients diagnosed with stage $3 / 4$ gastric cancer increase patients' acceptance of palliative care earlier in the cancer continuum?
}

\author{
Rhea Sharma ${ }^{1}$, Joseph McCollom² \\ ${ }^{1}$ Indiana University School of Medicine, ${ }^{2}$ Director of Palliative, Integrative and Survivorship \\ Oncology, Gastrointestinal Medical Oncology, Parkview Cancer Institute
}

\begin{abstract}
Background and Hypothesis: Palliative care, though designed improve quality of life in patients diagnosed with a grave illness, is widely stigmatized as an "end of life" service. Cancer diagnosis has been known to cause mental stress on patients; the added stigmatization of palliative care with death is a barrier to care, especially in gastric cancer patients whose delay in treatment can result in poor cancer prognosis and outcomes. Cognitive Behavior Therapy (CBT) intervention is promising is an ideal intervention in this case, yet, understudied in palliative oncology. Successful CBT intervention can combat guilt, denial and stigma, leading to more positive outlook on life and palliative care. We hypothesize that CBT intervention will increase stage 3/4 gastric cancer patients' acceptance to start palliative care early in their cancer treatment by decreasing associated stigma.
\end{abstract}

Experimental Design and Results: Study will be an interventional, unblinded, randomized clinical trial with an experimental and control group. A CBT video intervention will be used, preceded and followed by a pre-/post session questionnaire generated from IU's RedCap. Full intervention will have 4 sessions, with experimental groups divided into those receiving $<2,3$ or full 4 sessions. All individuals age 18+ with a recent stage $3 / 4$ gastric cancer diagnosis, who have the ability to provide consent/comprehend the CBT video will be illegible to participate in the study. A spearman's correlation will be used to test significance of CBT. A negative trend is predicted between CBT intervention and palliative care stigma; as the number of CBT sessions increase, palliative care stigma should decrease accordingly.

Potential Impact: CBT's effectiveness in alleviating stigma faced by patients with advanced gastric cancer can suggest more psychosocial training for physicians to move away from disease centered approach to one that implements ways that eradicate social barriers to care. 